\title{
Region-Specific Hierarchy between Atrophy, Hypometabolism, and $\beta$-Amyloid (A $\beta$ ) Load in Alzheimer's Disease Dementia
}

\author{
Renaud La Joie, ${ }^{1,2,3,4}$ Audrey Perrotin, ${ }^{1,2,3,4}$ Louisa Barré, ${ }^{6}$ Caroline Hommet, ${ }^{7,8,9}$ Florence Mézenge, ${ }^{1,2,3,4}$ \\ Méziane Ibazizene, ${ }^{6}$ Vincent Camus, ${ }^{7,8,10}$ Ahmed Abbas, ${ }^{1,2,3,4}$ Brigitte Landeau, ${ }^{1,2,3,4}$ Denis Guilloteau, ${ }^{7,8,11}$ \\ Vincent de La Sayette, ${ }^{1,2,3,5}$ Francis Eustache, ${ }^{1,2,3,4}$ Béatrice Desgranges, ${ }^{1,2,3,4}$ and Gaël Chételat ${ }^{1,2,3,4}$ \\ ${ }^{1}$ Institut National de la Santé et de la Recherche Médicale (Inserm), Unité 1077, ${ }^{2}$ Université de Caen Basse-Normandie, Unité Mixte de Recherche (UMR) \\ S1077, ${ }^{3}$ Ecole Pratique des Hautes Etudes, UMR-S1077, ${ }^{4}$ Unité 1077 and ${ }^{5}$ Service de Neurologie, Centre Hospitalier Universitaire de Caen, and ${ }^{6}$ Laboratoire \\ de Développements Méthodologiques en Tomographie par Emission de Positons, Commissariat à l'Energie Atomique/Division des Sciences de la \\ Vie/Institut d'Imagerie Biomédicale, Centre d'Imagerie Cérébrale et de Recherche en Neurosciences (Cyceron), 14000 Caen, France, ${ }^{7}$ Université François \\ Rabelais de Tours, UMR-S930 and Equipe de Recherche Labellisée 3106, ${ }^{8}$ Inserm, Unité 930, ${ }^{9}$ Centre Mémoire Ressources Recherche, ${ }^{10} \mathrm{Clinique}$ \\ Psychiatrique Universitaire, and ${ }^{11}$ Service de Médecine Nucléaire, Centre Hospitalier Régional et Universitaire de Tours, 37000 Tours, France
}

Gray matter atrophy, glucose hypometabolism, and $\beta$-amyloid $\mathrm{A} \beta$ deposition are well-described hallmarks of Alzheimer's disease, but their relationships are poorly understood. The present study aims to compare the local levels of these three alterations in humans with Alzheimer's disease. Structural magnetic resonance imaging, ${ }^{18} \mathrm{~F}$-fluorodeoxyglucose positron emission tomography (PET), and ${ }^{18} \mathrm{~F}$ florbetapir PET data from 34 amyloid-negative healthy controls and 20 demented patients with a high probability of Alzheimer's disease etiology (attested using neuroimaging biomarkers as recently recommended) were analyzed. For each patient and imaging modality, age-adjusted $Z$-score maps were computed, and direct between-modality voxelwise comparison and correlation analyses were performed. Significant differences in the levels of atrophy, hypometabolism, and A $\beta$ deposition were found in most brain areas, but the hierarchy differed across regions. A cluster analysis revealed distinct subsets of regions: (1) in the hippocampus, atrophy exceeded hypometabolism, whereas $\mathrm{A} \beta$ load was minimal; (2) in posterior association areas, $\mathrm{A} \beta$ deposition was predominant, together with high hypometabolism and lower but still significant atrophy; and (3) in frontal regions, A $\beta$ deposition was maximal, whereas structural and metabolic alterations were low. Atrophy and hypometabolism significantly correlated in the hippocampus and temporo-parietal cortex, whereas $\mathrm{A} \beta$ load was not significantly related to either atrophy or hypometabolism. These findings provide direct evidence for regional variations in the hierarchy and relationships between $\mathrm{A} \beta$ load, hypometabolism, and atrophy. Altogether, these variations probably reflect the differential involvement of region-specific pathological or protective mechanisms, such as the presence of neurofibrillary tangles, disconnection, as well as compensation processes.

\section{Introduction}

Alzheimer's disease is characterized by major brain changes, including gray matter atrophy, hypometabolism, and $\beta$-amyloid $(\mathrm{A} \beta)$ deposition. These changes have been described extensively using neuroimaging techniques, i.e., magnetic resonance imaging (MRI)

\footnotetext{
Received May 4, 2012; revised Aug. 2, 2012; accepted Sept. 3, 2012.

Author contributions: R.L.J., V.d.L.S., F.E., B.D., and G.C. designed research; R.L.J., A.P., F.M., V.d.L.S., F.E., B.D., and G.C. performed research; L.B., C.H., M.I., V.C., A.A., B.L., and D.G. contributed unpublished reagents/analytic tools; R.L.J., F.M., and B.L. analyzed data; R.L.J. and G.C. wrote the paper.

This study was funded by Agence Nationale de la Recherche (Longévité et Vieillissement 2007), Programme Hospitalier de Recherche Clinique (National 2008), Région Basse Normandie, and Institut National de la Santé et de la Recherche Médicale (Inserm), including the Inserm Liliane Bettencourt School (MD-PhD Program). We are grateful to M. Fouquet and N. Villain for their comments and support. We thank K. Mevel, H. Mirabel, M. Gaubert, S. Egret, A. Pélerin, C. Schupp, J. Dayan, A. Quillard, A. Manrique, C. Lebouleux, M. H. Noel, and M. C. Onfroy for their help in the data acquisition and the volunteers who participated in this study.

Correspondence should be addressed to Dr. Gaël Chételat, Unité de Recherche U1077, Centre Cyceron, Boulevard H. Becquerel, Boîte Postale 5229, 14074 Caen, France. E-mail: chetelat@cyceron.fr.

DOI:10.1523/JNEUROSCI.2170-12.2012

Copyright $\odot 2012$ the authors $\quad 0270-6474 / 12 / 3216265-09 \$ 15.00 / 0$
}

and positron emission tomography (PET) combined with specific radiotracers: ${ }^{18} \mathrm{~F}$-fluorodeoxyglucose (FDG) for glucose metabolism, ${ }^{11} \mathrm{C}$-Pittsburgh compound $\mathrm{B}$ and ${ }^{18} \mathrm{~F}$-florbetapir or others for $\mathrm{A} \beta$ deposition (Herholz and Ebmeier, 2011). These techniques have proved to be useful for early diagnosis of Alzheimer's disease but also for additional understanding the pathological mechanism(s) underlying the disease (Rabinovici and Roberson, 2010).

Dynamic models of changes in these neuroimaging biomarkers over the course of the disease have been proposed recently (Perrin et al., 2009; Jack et al., 2010; Ewers et al., 2011). According to the amyloid cascade hypothesis (Hardy and Selkoe, 2002), the sequence of events would start with $\mathrm{A} \beta$ deposition in a very early, presymptomatic stage. Then, $A \beta$ is thought to trigger functional and structural changes that would appear latter, closer to clinical symptom onset. However, despite experimental studies showing that $\mathrm{A} \beta$ impairs synaptic functioning (Palop and Mucke, 2010; Parihar and Brewer, 2010), results from the human neuroimaging literature are ambiguous. Correlations between increased $\mathrm{A} \beta$ 

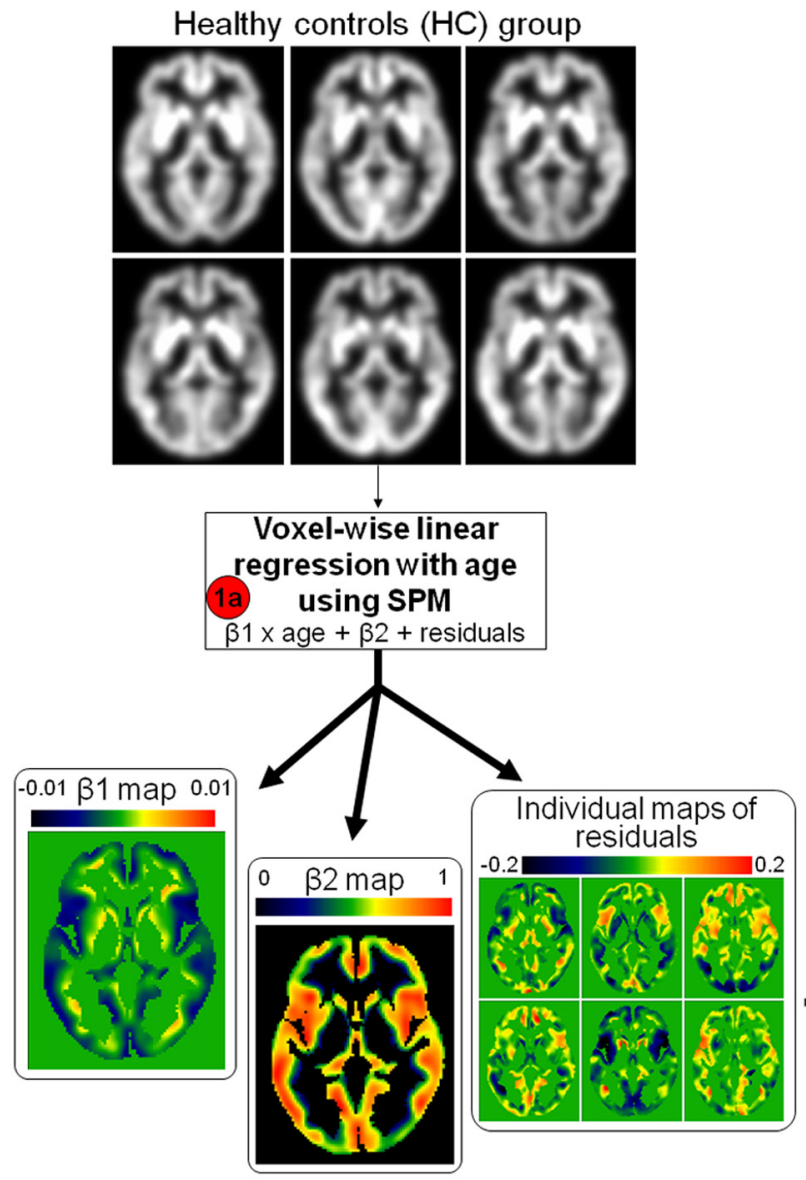

One patient with Alzheimer's disease $($ age $=65)$

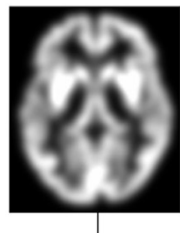

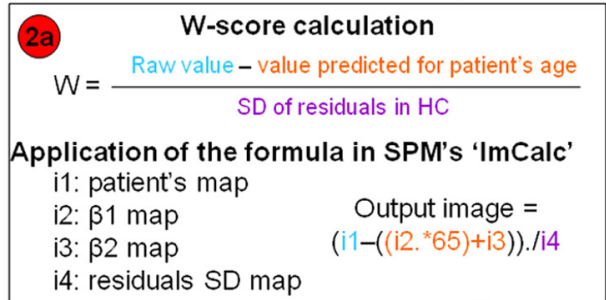
i4: residuals SD map

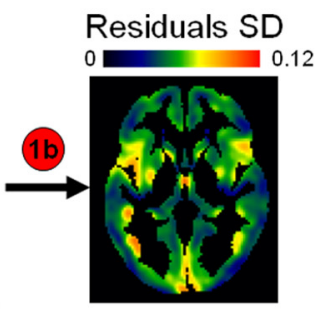

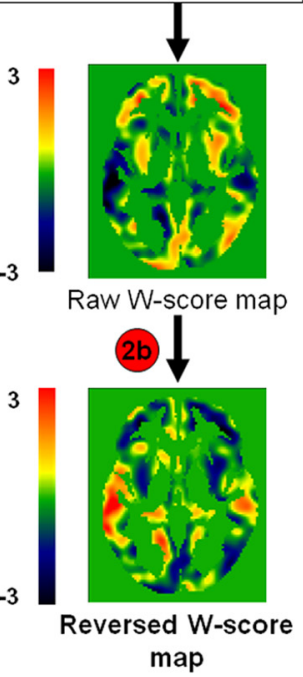

Figure 1. Illustration of the different steps required to create W-score maps using the SPM software. This figure shows the procedure for the atrophy W-score map of a 65 -year-old patient with Alzheimer's disease compared with six healthy controls $(\mathrm{HC})$ for the sake of illustration. First, a simple linear regression was performed in the HC group to estimate age-related changes ( 1 a), resulting in several files: the $\beta 1$ map containing voxelwise age-related regression coefficients, the $\beta 2$ map containing intercept values, and the individual maps of residuals. The SD of residuals was computed voxelwise (1b). A W-score map was then created using the corresponding formula and previously computed maps using the SPM "ImCalc" function (2a). Last, for MRI and FDG-PET data, W values were reversed so that positive numbers represent pathological features in all three imaging modalities $(\mathbf{2} \boldsymbol{b})$.

and decreased metabolism in Alzheimer's disease patients have been found in some studies (Engler et al., 2006; Edison et al., 2007; Cohen et al., 2009) but not in others (Li et al., 2008; Rabinovici et al., 2010; Furst et al., 2012). Previous works showed that the relationship between $\mathrm{A} \beta$ load and hypometabolism (Cohen et al., 2009) or atrophy (Chételat et al., 2010) varies throughout the evolution of the disease, suggesting that neurodegeneration could be independent of $\mathrm{A} \beta$ pathology in later stages (Hyman, 2011).

Moreover, there may also be differences in the sequence of events according to brain regions, as suggested by discrepancies in the regional pattern of atrophy, hypometabolism, and $\mathrm{A} \beta$ deposition. More specifically, although all three alterations are found in some regions, such as the posterior cingulate, precuneus, and temporo-parietal areas (Buckner et al., 2005; Jack et al., 2008), regional discrepancies have been highlighted when comparing modalities two by two. Thus, differential degrees of atrophy and hypometabolism are found in the posterior cingulate cortex versus the hippocampus, for example (Alsop et al., 2008; Chételat et al., 2008), and both atrophy and hypometabolism can be found in regions with low $\mathrm{A} \beta$ load or can be absent in regions with high A $\beta$ deposition (Edison et al., 2007; Jack et al., 2008; Li et al., 2008).
The main objective of this study was therefore to characterize and compare the regional degrees of gray matter atrophy, hypometabolism, and $\mathrm{A} \beta$ deposition as measured in the same patients with Alzheimer's disease. In addition, we aimed at assessing for the first time the local correlations between these three alterations using a voxel-based method. For these purposes, we thoroughly selected patients as having a high probability of Alzheimer's disease etiology according to recent recommendations (McKhann et al., 2011) and used a methodology especially designed to compare data from different imaging modalities (Chételat et al., 2008).

\section{Materials and Methods}

\section{Participants}

Twenty-two patients diagnosed with probable Alzheimer's disease were first selected according to National Institute of Neurological and Communicative Diseases and Stroke/Alzheimer's Disease and Related Disorders Association clinical criteria (McKhann et al., 1984). Following the recent recommendations from the National Institute on Aging and Alzheimer's Association workgroup (McKhann et al., 2011) for research studies, only those 20 patients with a high probability of Alzheimer's disease etiology (i.e., who have positive neuroimaging biomarkers for both $\mathrm{A} \beta$ deposition and neurodegeneration) were selected for additional analyses (see below). 
Table 1. Selection of patients with a high probability of Alzheimer's disease etiology based on neuroimaging biomarkers of both amyloid deposition and neuronal degeneration (McKhann et al., 2011)

\begin{tabular}{|c|c|c|c|c|c|c|c|c|c|c|c|c|c|c|c|c|c|c|c|c|c|c|c|}
\hline \multirow[b]{2}{*}{ Type of biomarker } & \multirow[b]{2}{*}{ Index used } & \multicolumn{22}{|c|}{ Patients with a clinical diagnosis of probable Alzheimer's disease } \\
\hline & & 1 & 2 & 3 & 4 & 5 & 6 & 7 & 8 & 9 & 10 & 11 & 12 & 13 & 14 & 15 & 16 & 17 & 18 & 19 & 20 & 21 & 22 \\
\hline$A \beta$ deposition & $\begin{array}{l}\text { Florbetapir neocortical } \\
\text { SUVr }\end{array}$ & 1.46 & 1.54 & 1.86 & 1.20 & 1.48 & 1.81 & 0.87 & 1.80 & 1.92 & 1.71 & 1.41 & 1.77 & 1.56 & 1.28 & 1.29 & 1.83 & 1.72 & 0.89 & 1.59 & 1.68 & 1.45 & 1.76 \\
\hline Neuronal degeneration & $\begin{array}{l}\text { Atrophy W-score } \\
\text { Hypometabolism } \\
\text { W-score }\end{array}$ & $\begin{array}{l}3.36 \\
1.39\end{array}$ & $\begin{array}{l}1.82 \\
4.44\end{array}$ & $\begin{array}{l}5.71 \\
4.88\end{array}$ & $\begin{array}{l}1.33 \\
1.75\end{array}$ & $\begin{array}{l}3.52 \\
5.51\end{array}$ & $\begin{array}{l}4.77 \\
5.83\end{array}$ & $\begin{array}{l}6.11 \\
1.96\end{array}$ & $\begin{array}{l}3.24 \\
3.26\end{array}$ & $\begin{array}{l}2.37 \\
3.26\end{array}$ & $\begin{array}{l}2.07 \\
4.33\end{array}$ & $\begin{array}{l}1.36 \\
2.97\end{array}$ & $\begin{array}{l}2.51 \\
4.42\end{array}$ & $\begin{array}{l}0.13 \\
1.73\end{array}$ & $\begin{array}{l}1.08 \\
1.89\end{array}$ & $\begin{array}{l}2.84 \\
3.05\end{array}$ & $\begin{array}{l}1.91 \\
5.52\end{array}$ & $\begin{array}{l}4.25 \\
4.83\end{array}$ & $\begin{array}{l}2.01 \\
2.30\end{array}$ & $\begin{array}{l}1.72 \\
2.40\end{array}$ & $\begin{array}{l}2.85 \\
4.84\end{array}$ & $\begin{array}{l}3.80 \\
1.62\end{array}$ & $\begin{array}{l}1.38 \\
4.30\end{array}$ \\
\hline $\begin{array}{c}\text { Presence of both } \\
\text { biomarkers? }\end{array}$ & & Yes & Yes & Yes & Yes & Yes & Yes & No & Yes & Yes & Yes & Yes & Yes & Yes & Yes & Yes & Yes & Yes & No & Yes & Yes & Yes & Yes \\
\hline
\end{tabular}

Thirty-seven healthy controls were also enrolled in this study after clinical and neuropsychological examination. They had no history or clinical evidence of major neurological or psychiatric disorder and performed in the normal range in all neuropsychological tests (including tests of episodic memory, working memory, language skills, executive functions, and visuospatial abilities).

The study was approved by the local ethics committee, and all participants gave written consent for participation before the scans.

\section{Neuroimaging data acquisition}

All participants were scanned on the same MRI and PET cameras at the Cyceron Centre (Caen, France). The median time lapse between the first and last examination was $16.5 \mathrm{~d}$ (minimum of 1 ; maximum of 60 ) for patients and $28 \mathrm{~d}$ (minimum of 8 ; maximum of 215) for controls.

MRI data. For each participant, a high-resolution T1-weighted anatomical image was acquired on a Philips Achieva $3 \mathrm{~T}$ scanner using a three-dimensional fast-field echo sequence (sagittal; repetition time, 20 ms; echo time, $4.6 \mathrm{~ms}$; flip angle, $20^{\circ}$; 170 slices; slice thickness, $1 \mathrm{~mm}$; field of view, $256 \times 256 \mathrm{~mm}^{2}$; matrix, $256 \times 256$ ).

PET data. Both FDG and florbetapir PET scans were acquired on a Discovery RX VCT 64 PET-CT device (GE Healthcare) with a resolution of $3.76 \times 3.76 \times 4.9 \mathrm{~mm}$ (field of view, $157 \mathrm{~mm}$ ). Forty-seven planes were obtained with a voxel size of $2.7 \times 2.7 \times 3.27 \mathrm{~mm}$. A transmission scan was performed for attenuation correction before the PET acquisition.

$F D G-P E T$. Participants were fasted for at least $6 \mathrm{~h}$ before scanning. After a 30 min resting period in a quiet and dark environment, $\sim 180$ MBq of FDG was intravenously injected as a bolus. A 10 min PET acquisition scan began 50 min after injection.

Florbetapir-PET. Each participant underwent a $20 \mathrm{~min}$ PET scan, beginning $50 \mathrm{~min}$ after the intravenous injection of $\sim 4 \mathrm{MBq} / \mathrm{kg}$ of florbetapir.

\section{Neuroimaging data handling and transformation}

Preprocessing. MRI data were segmented, normalized, and modulated using the VBM5.1 toolbox (http://dbm.neuro.uni-jena.de), implemented in the Statistical Parametric Mapping 5 (SPM) software (Wellcome Trust Centre for Neuroimaging, London, UK) to obtain maps of local gray matter volume corrected for brain size. PET data (both FDG and florbetapir) were corrected for partial volume effects (PMOD Technologies), coregistered onto their corresponding MRI, and normalized using the deformation parameters defined from the MRI procedure. Resultant images underwent quantitative scaling using the cerebellar gray matter as a reference to obtain standardized uptake value ratio (SUVr) images.

Because MRI and PET data have different original spatial resolutions, a differential smoothing was applied to equalize the effective smoothness (Richardson et al., 1997; Chételat et al., 2008; Villain et al., 2008): a Gaussian kernel of $10 \times 10 \times 10(x, y, z) \mathrm{mm}$ was used for the MRI data and $9.3 \times 9.3 \times 8.8 \mathrm{~mm}$ for the PET data. Finally, images were masked to exclude non-gray matter voxels from the analyses.

Creation of $\mathrm{W}$-score maps. To obtain measurements of atrophy, hypometabolism, and amyloid load expressed in the same unit, therefore
Table 2. Demography and mini-mental state examination scores in the groups used for data analyses

\begin{tabular}{lccr}
\hline & $\mathrm{AD}(n=20)$ & $\mathrm{HC}(n=34)$ & $p$ value \\
\hline Age (years) & $68.9 \pm 9.0$ & $68.1 \pm 7.2$ & 0.83 \\
Women (\%) & $10(50.0 \%)$ & $20(58.8 \%)$ & 0.73 \\
Education (years) & $10.35 \pm 3.8$ & $11.8 \pm 3.7$ & 0.11 \\
MMSE & $20.6 \pm 4.5$ & $29.1 \pm 0.8$ & $<0.001$ \\
\hline
\end{tabular}

Statistical analyses were performed using Mann-Whitney $U$ test [ for age, education, and mini-mental state examination (MMSE)] and Yates's $\chi^{2}$ test (sex ratio). AD, Alzheimer's disease patients; $H C$, healthy controls.

enabling a direct comparison of different imaging modalities, $W$-score maps were computed for each patient and each imaging modality using the healthy control group as a reference (Fig. 1). $W$-scores are analogous to $Z$-scores but they are adjusted for specific covariate(s) (Jack et al., 1997; Boccardi et al., 2003; Jack et al., 2008), age in the present case. Like $Z$-scores, $W$-scores have a mean value of 0 and an SD of 1 in the control group, and values of +1.65 and -1.65 correspond to the 95 th and 5 th percentiles, respectively. To create $W$-score maps, voxelwise regressions were first performed in the control group between age and each imaging data using the SPM software. Then, $W$-score maps was computed using the following formula: $W$-score $=[$ (patient's raw value $)-($ value expected in the control group for the patient's age)]/SD of the residuals in controls.

For MRI and FDG-PET, $W$-scores were reversed so that positive $W$-scores indicate pathology for all modalities (i.e., less gray matter volume, less glucose metabolism, more $\mathrm{A} \beta$ deposition). For each modality, individual $W$-score maps were averaged across the patients to provide whole-brain profiles of atrophy, hypometabolism, and $\mathrm{A} \beta$ deposition, expressed as mean $W$-scores.

Regions of interest. Finally, to obtain quantitative and statistical information in specific brain areas, mean values of gray matter volume, FDG SUVr, and florbetapir SUVr were extracted from the corresponding images before the smoothing step in 11 regions of interest defined using the automated anatomical labeling atlas (Tzourio-Mazoyer et al., 2002): hippocampus, amygdala, parahippocampus, temporal pole, angular gyrus, precuneus/posterior cingulate, lateral temporal, anterior cingulate, orbitofrontal, dorsomedial prefrontal, and dorsolateral prefrontal cortices. The neocortical values of florbetapir retention were also extracted from the florbetapir SUVr images for the sake of classification of patients as amyloid positive versus negative (see below).

Selection of patients and subjects using neuroimaging biomarkers Patients. As mentioned above, neuroimaging biomarkers were used to further select those Alzheimer's disease patients with a high likelihood of Alzheimer's disease etiology, i.e., showing positive biomarkers of both $\mathrm{A} \beta$ deposition (from florbetapir-PET) and neurodegeneration (from MRI or FDG-PET), as recommended (McKhann et al., 2011). First, values of florbetapir neocortical SUVr were used to separate amyloidpositive from amyloid-negative patients. For that purpose, a florbetapir SUVr cutoff value was determined using a cluster analysis performed on 


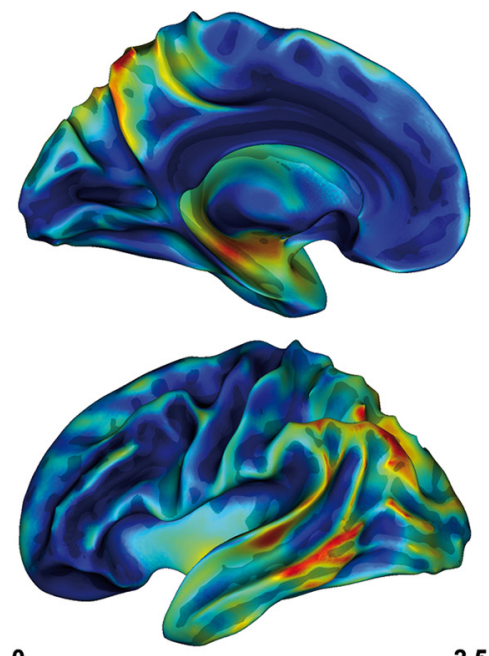
ATROPHY W-SCORE
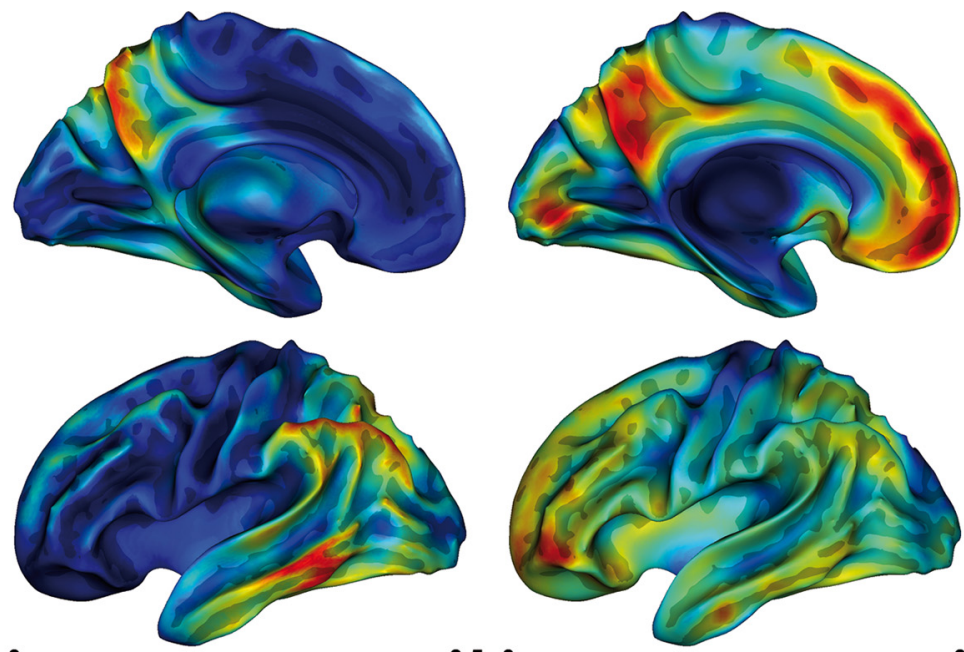

3.50 HYPOMETABOLISM W-scORE

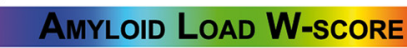

Figure 2. Brain patterns of alteration in the 20 patients with Alzheimer's disease dementia. For each imaging modality, local degrees of alteration are expressed as mean $W$-score compared with the control group $(n=34)$ in each gray matter voxel. Note that, for all imaging modalities, a positive $W$-score indicates a pathological feature. Colors have been scaled to the range of each modality to fit to the regional distribution of each process. For clarity, only the left hemisphere is represented here, because results were mainly symmetrical.

the whole sample (Bourgeat et al., 2010; Rowe et al., 2010), yielding to a value of 1.1. This value is consistent with previous studies using florbetapir (Fleisher et al., 2011; Camus et al., 2012).

Then, neurodegeneration was assessed using two specific indexes. For atrophy, the hippocampus, amygdala, and lateral temporal cortex were combined, and a global $W$-score was computed. Hypometabolism $W$-score included the angular gyrus, precuneus, and posterior cingulate. These areas were chosen as the prototypical neural substrates of Alzheimer's disease, according to a recent meta-analyses of MRI and FDG-PET studies (Schroeter et al., 2009).

Patients were included if they had a positive florbetapir-PET scan and at least one positive biomarker of neurodegeneration ( $W$-score $\geq 1.65$ for atrophy and/or hypometabolism). Individual values for the three biomarkers are shown in Table 1 . Of the 22 clinically diagnosed patients, two showed a negative florbetapir-PET scan. For the 20 remaining patients, at least one of the two biomarkers of neuronal injury was positive. Consequently, these 20 Alzheimer's disease patients were all included in the following analyses. Only three of these 20 patients were treated with cholinesterase inhibitors when included, whereas the others were de novo (untreated) patients. All analyses were repeated without these three patients, and the conclusions remained unchanged (data not shown) so that all results will be presented on the complete sample of 20 patients.

Healthy controls. In addition, to avoid the presence of healthy subjects at a preclinical stage of Alzheimer's disease in the control group that could bias our analyses, amyloid-positive subjects were excluded from additional analysis. Indeed, the presence of cerebral amyloidosis is believed to indicate the first stage of preclinical Alzheimer's disease (Sperling et al., 2011). Therefore, three subjects with a florbetapir SUVr value $>1.1$ were withdrawn from the control group.

Demographic data and mini-mental state examination scores for the 34 controls ( 14 males, 20 females) and 20 patients with highly probable Alzheimer's disease (10 males, 10 females) are displayed in Table 2.

\section{Statistical analyses}

Comparing local atrophy, hypometabolism, and $A \beta$ burden: voxelwise analyses. Individual $W$-score maps of atrophy, hypometabolism, and $\mathrm{A} \beta$ deposition were compared in a voxelwise factorial analysis using the SPM software, with MRI, FDG-PET, and florbetapir-PET images as withinsubject measurements. Results were considered as significant at $p$ [familywise error (FWE) corrected for multiple comparisons] $<0.05$ threshold and cluster size $k \geq 20\left(160 \mathrm{~mm}^{3}\right)$.

Regions of interest analyses. Using the values extracted from the 11 regions of interest, regional $W$-scores were calculated and compared us- ing nonparametric statistical tests. To compare the local degrees of atrophy, hypometabolism, and A $\beta$ deposition, a Friedman's ANOVA was performed within each region of interest. If significant (i.e., $p<0.05$ ), post hoc two-by-two comparisons were conducted using Wilcoxon's signed-rank test. So as to provide a more global insight on the different gradients of alteration across brain regions, a Ward's hierarchical clustering method was used to group brain regions with closely similar patterns.

Correlation between local alterations. To assess the link between local atrophy, hypometabolism, and $\mathrm{A} \beta$ deposition, $W$-score maps were entered two by two in voxelwise correlation analyses using the Biological Parametric Mapping toolbox implemented in SPM and especially designed to analyze brain images from different modalities (Casanova et al., 2007). Three independent correlation analyses were performed: $W_{\mathrm{A} \beta}$ versus $W_{\text {hypometabolism }}$ $W_{\mathrm{A} \beta}$ versus $W_{\text {atrophy }}$, and $W_{\text {atrophy }}$ versus $W_{\text {hypometabolism. }}$. Results were considered as significant at a $p_{\mathrm{FWE}}<0.05$ threshold and cluster size $k \geq 20(160$ $\left.\mathrm{mm}^{3}\right)$.

\section{Results}

Patterns of gray matter atrophy, hypometabolism, and A $\beta$ deposition in Alzheimer's disease

Averaged $W$-score maps for each modality are shown in Figure 2. Briefly, gray matter atrophy was found in the medial and lateral temporal, inferior parietal cortex, and precuneus. Hypometabolism concerned the precuneus, posterior cingulate, lateral temporal and parietal cortices, and, to a lesser degree, the medial temporal lobe. $\mathrm{A} \beta$ deposition was found in most brain areas, with highest $W$-scores in the medial and orbital prefrontal cortex, precuneus, and posterior cingulate (with $W$-score $\geq 9$ ), whereas the primary sensorimotor cortex, occipital cortex, thalamus, and medial temporal lobe were relatively spared.

\section{Voxelwise comparisons between alterations}

The results of the direct between-modality comparison analyses are displayed in Figure 3. First, the comparison between atrophy and hypometabolism revealed significant differences in both directions. Hypometabolism was greater than atrophy in the precuneus, posterior cingulate, parietal, and middle/inferior temporal gyrus, whereas atrophy significantly exceeded hypometabolism in the anterior part of medial temporal lobe. Second, $\mathrm{A} \beta$ 

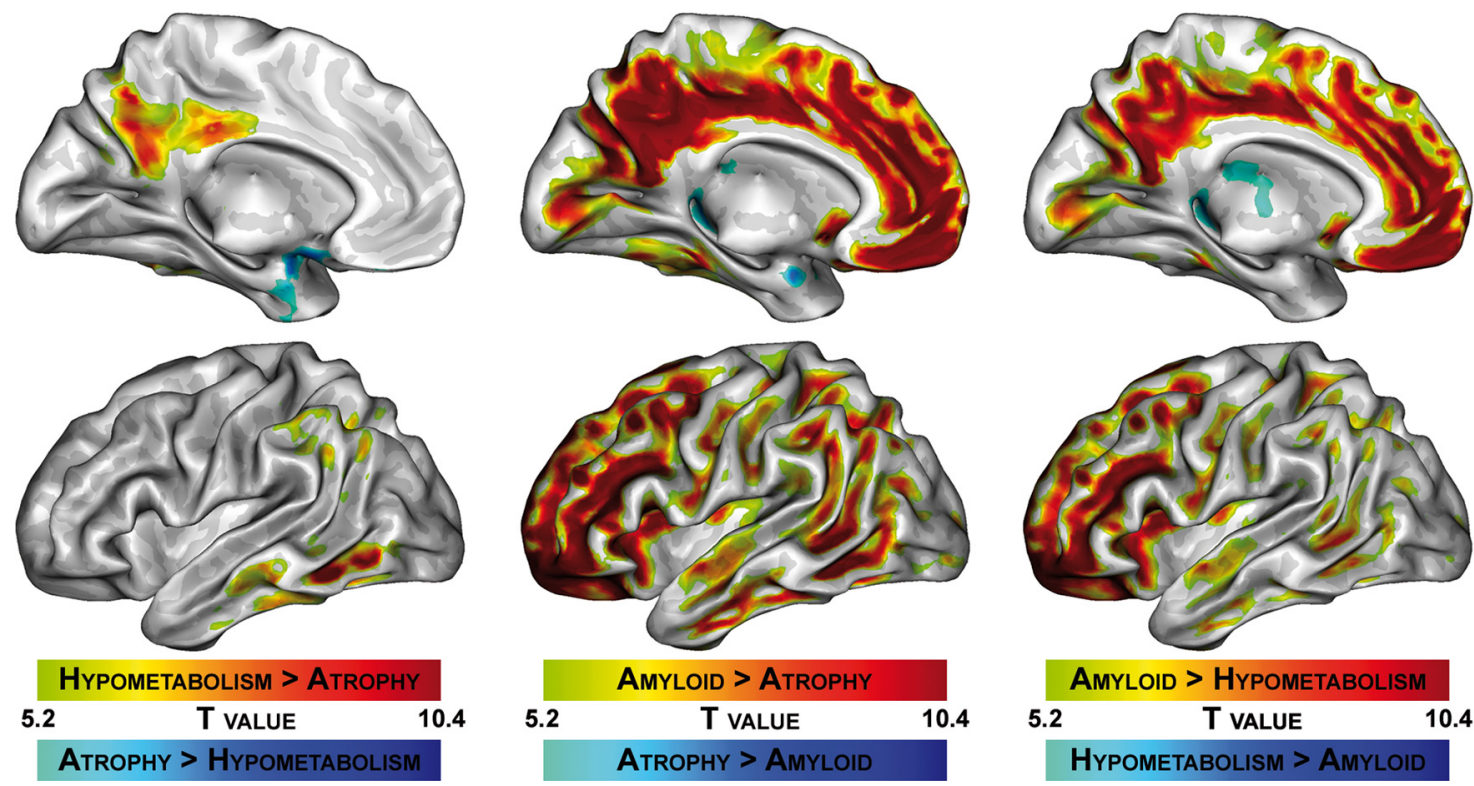

Figure 3. Voxelwise comparisons between the local degrees of atrophy, hypometabolism, and $A \beta$ deposition in the 20 patients with Alzheimer's disease dementia. The $t$ value of 5.2 used as a threshold in this figure corresponds to the $p$ (FWE corrected) $<0.05$ threshold described in Results.

deposition significantly exceeded atrophy in most brain areas, with greatest differences in frontal areas, precuneus, and posterior cingulate and less but still significant differences in lateral temporal and parietal regions. Conversely, atrophy was superior to $\mathrm{A} \beta$ deposition in the anterior part of the medial temporal lobe and in the posterior hippocampus. Third, $\mathrm{A} \beta$ deposition considerably exceeded hypometabolism in prefrontal cortex, precuneus, and posterior cingulate, whereas the difference was less marked, but still significant, in the lateral temporal and parietal cortices. Finally, hypometabolism significantly exceeded A $\beta$ deposition in the thalamus and posterior hippocampus.

\section{Voxelwise correlations between alterations}

The two-by-two intermodality correlation analyses revealed significant results for the atrophy versus hypometabolism analysis only (Fig. 4). Specifically, hypometabolism was correlated to local atrophy in the hippocampus, temporo-parietal cortex, cuneus/precuneus junction, as well as the dorsolateral prefrontal cortex. In contrast, correlations between $\mathrm{A} \beta$ deposition and atrophy or hypometabolism did not reach significance at the $p_{\mathrm{FWE}}<0.05$ threshold. Using a more permissive statistical threshold of $p_{\text {uncorrected }}<0.001$, a significant positive correlation was found between $\mathrm{A} \beta$ load and hypometabolism in a small cluster within the retrosplenial cortex/posterior cingulate (MNI coordinates at the peak: $-4,-52,16 ; t$ value $=4.53$; cluster size $=14$ voxels).

\section{Regions of interest analyses}

The results of the ANOVA comparing the regional $W$-scores of the three alterations within the regions of interest are displayed in Figure 5. Significant differences were found between the degrees of atrophy, hypometabolism, and $\mathrm{A} \beta$ deposition in all investigated areas, except for the amygdala (Friedman's ANOVA did not reach significance, $p=0.55$ ). Post hoc comparisons performed with Wilcoxon's signed-rank test showed clearly different gradients according to regions (Fig. 5), with areas of predominant atrophy (such as the hippocampus) and areas of excessive $\mathrm{A} \beta$ deposition compared with atrophy and hypometabolism (such as the prefrontal cortex).
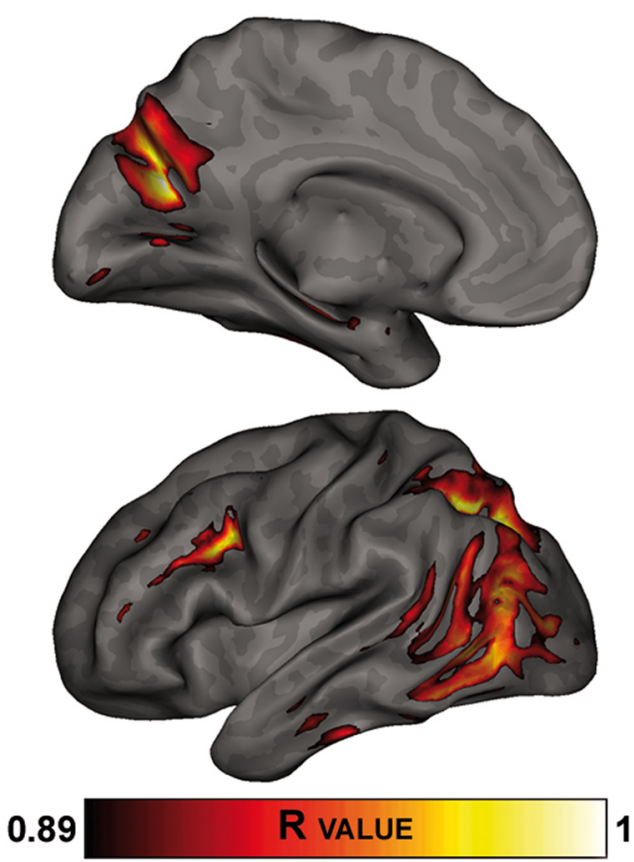

Figure 4. Voxelwise correlations between local degrees of atrophy and hypometabolism in the 20 patients with Alzheimer's disease dementia. The $R$ value of 0.89 used as a threshold in this figure corresponds to the $p$ (FWE corrected) $<0.05$ threshold described in Results.

Using the three mean $W$-scores of each region, Ward's hierarchical clustering method led to the distinction between four subsets of brain areas (Fig. 6, left). A repeated-measures ANOVA was conducted on the mean $W$-score values from the four sets of regions, with two factors: brain region (with four levels) and imaging modality (with three levels). It revealed a highly significant region $\times$ modality interaction $\left(F_{(6,152)}=35.0 ; p<10^{-5}\right)$, which confirmed the differential hierarchy between the levels of the three markers in these four sets of brain regions (Fig. 6, right). In the hippocampo-amygdala complex, atrophy was marked 


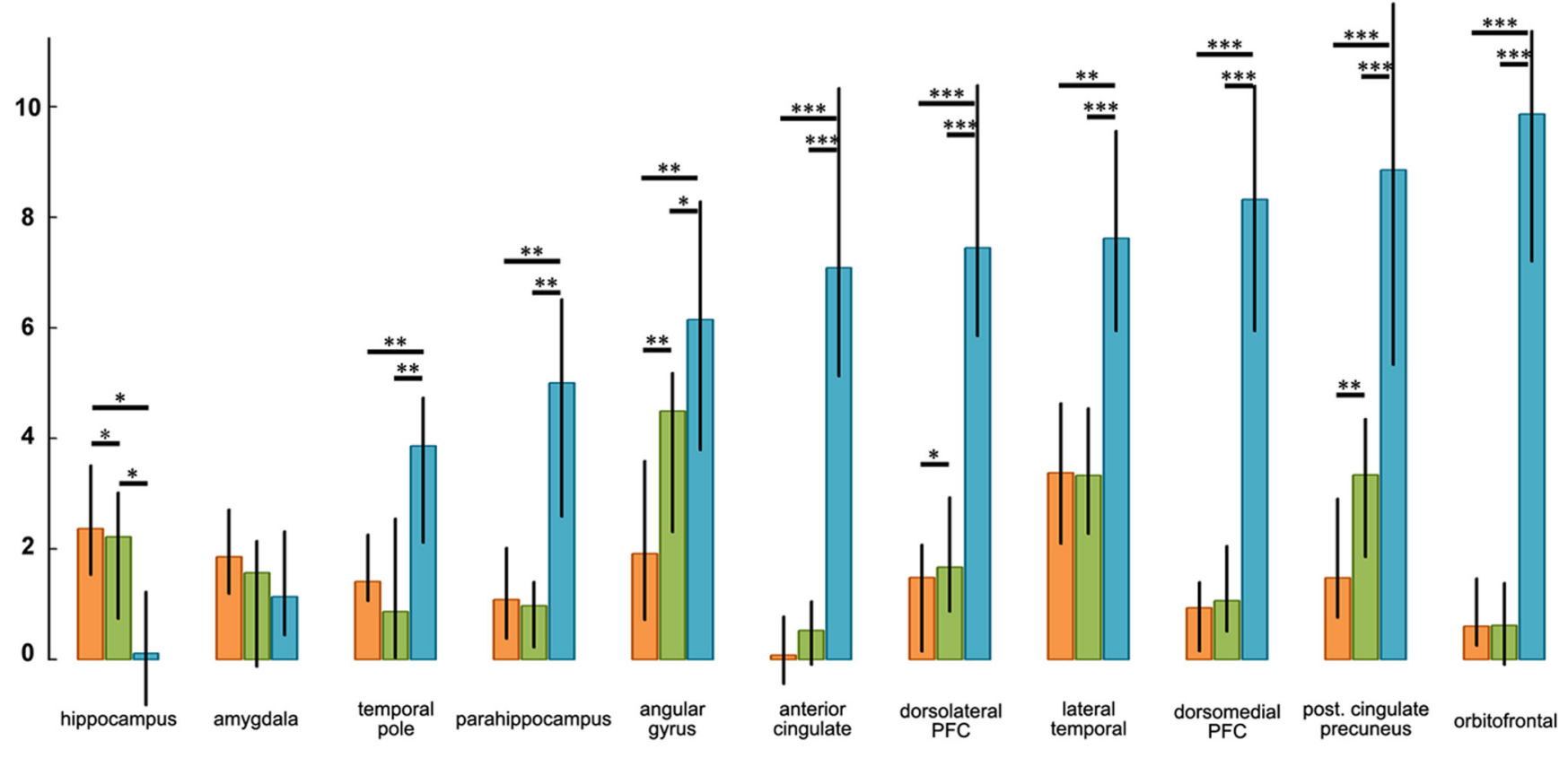

Figure 5. Regions of interest analyses. Local degrees of atrophy (orange), hypometabolism (green), and A $\beta$ deposition (blue) expressed as mean $W$-scores were compared using Friedman's ANOVA. When significant $(p<0.05)$, post hoc analyses were performed using Wilcoxon's test $\left({ }^{*} p<0.05 ;{ }^{* *} p<0.005 ;{ }^{* * *} p<0.0001\right)$. Histograms represent median values, and error bars refer to the interquartile range. Regions presented here are ordered by increasing amyloid $W$-score. post. cingulate, Posterior cingulate cortex; PFC, prefrontal cortex.

(median $W=2.16)$ and significantly exceeded hypometabolism $(W=1.99)$ and $\mathrm{A} \beta$ load $(W=0.50)$. In the parahippocampus and temporal pole, $\mathrm{A} \beta$ load was relatively high $(W=4.5)$ and exceeded local degrees of atrophy $(W=1.45)$ and hypometabolism $(W=0.78)$. In posterior association areas (lateral temporal cortex, angular gyrus, and precuneus/posterior cingulate), all modalities showed high to very high $W$-scores, with a predominance of $\mathrm{A} \beta$ deposition ( $W=7.36$ ), exceeding local hypometabolism $(W=3.69)$, itself exceeding atrophy ( $W=2.31)$. Last, in frontal areas (dorsolateral prefrontal cortex, dorsomedial prefrontal cortex, orbitofrontal cortex, and anterior cingulate), extreme $\mathrm{A} \beta$ deposition $(W=7.99)$ contrasted to the weakness of structural and metabolic defects ( 0.61 and 0.85 , respectively).

\section{Discussion}

In this study, three major brain alterations in Alzheimer's disease, namely gray matter atrophy, hypometabolism, and $\mathrm{A} \beta$ deposition, were measured concomitantly in the same patients with Alzheimer's disease dementia. Their relative expression and relationships were assessed both regionally and voxelwise. We showed marked regional variability in the hierarchy between these different brain alterations with three main profiles: (1) extreme $\mathrm{A} \beta$ deposition with low hypometabolism and atrophy (frontal areas); (2) predominance of $\mathrm{A} \beta$ deposition, together with high hypometabolism and lower but still significant atrophy (posterior association areas); and (3) predominance of atrophy and hypometabolism faced to low $\mathrm{A} \beta$ burden (in medial temporal areas). In addition, local correlations were found between atrophy and hypometabolism within a large parietotemporal network, whereas $\mathrm{A} \beta$ deposition did not correlate to either atrophy or hypometabolism.

One of the most striking results of this study is the considerable discrepancy between $A \beta$ deposition on the one hand and atrophy and hypometabolism on the other hand in patients with Alzheimer's disease dementia. First, the brain distribution of A $\beta$ deposition clearly differed from that of the two other alterations
(Fig. 3, middle and right). Second, $\mathrm{A} \beta$ deposition did not correlate to the other alterations. Previous studies assessing the correlations between $A \beta$ deposition and metabolism in patients with Alzheimer's disease reported conflicting results, and, when significant, correlations were only found in restricted areas, mostly in temporal and parietal cortices (Engler et al., 2006; Edison et al., 2007; Cohen et al., 2009; Forsberg et al., 2010). This is consistent with our finding of a restricted correlation in the posterior cingulate cortex when using a more permissive threshold. As for the link between atrophy and $\mathrm{A} \beta$ deposition, although relationships have been reported in preclinical stages, most studies reported no significant correlations in demented patients (Fagan et al., 2009; Chételat et al., 2010).

These findings are in line with the idea that, at symptomatic stages of the disease, $\mathrm{A} \beta$ does not play a prominent role in ongoing neurodegeneration (Hyman, 2011). Instead, tau pathology, disturbance in axonal transport, or inflammation processes could contribute to brain atrophy and metabolic disruption (see below). They could also be interpreted in the light of current models suggesting a time decay between the appearance of $\mathrm{A} \beta$ plaques, more than one decade before the first symptoms, and hypometabolism and atrophy thought to appear years later, closer to cognitive decline (Jack et al., 2010). Another interesting hypothesis is the possibility of distant, rather than local, effects of A $\beta$. Notably, Cohen et al. (2009) observed that metabolism in the precuneus of amyloid-positive patients with Alzheimer's disease was inversely correlated to $\mathrm{A} \beta$ deposition in frontal areas. Bourgeat et al. (2010) also reported a distant relationship (between temporal neocortical $\mathrm{A} \beta$ deposition and hippocampal atrophy), yet the correlation was observed in the cognitively normal group and not in demented patients.

In contrast, atrophy and hypometabolism were found in the same brain areas and were highly correlated (Fig. 4), suggesting that both alterations share at least partly common underlying mechanisms. The direct comparison between both processes also 


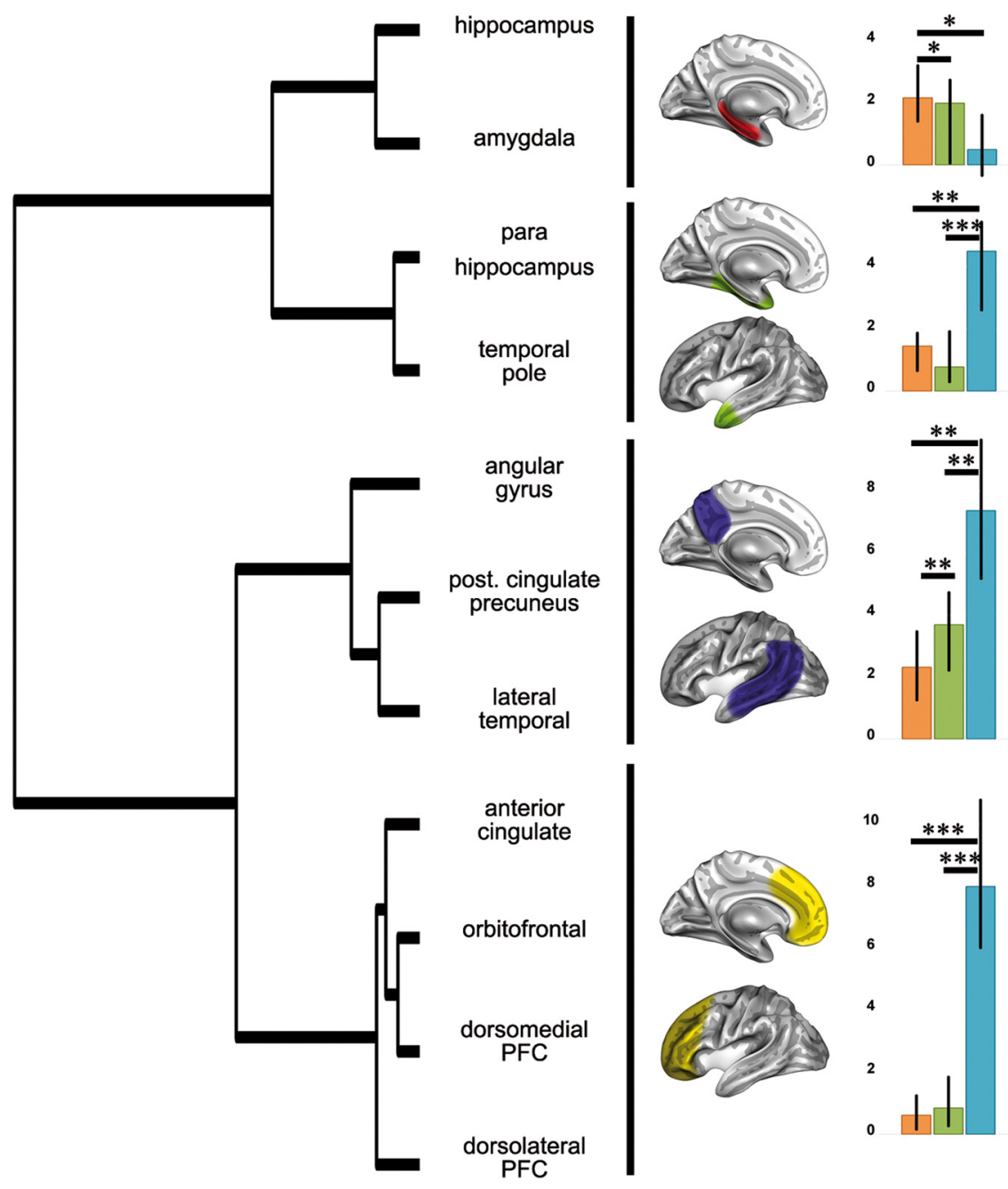

Figure 6. Classification of brain regions according to their degrees of atrophy, hypometabolism, and A $\beta$ deposition. Left, Ward's hierarchical clustering analysis performed on the regions of interest distinguished four subsets of brain areas according to their three mean $W$-scores. Right, For each subset, degrees of atrophy (orange), hypometabolism (green), and A $\beta$ load (blue) were averaged and compared using Friedman's ANOVA and Wilcoxon's test $\left({ }^{*} p<0.05 ;{ }^{* *} p<0.005 ;{ }^{* * *} p<0.0001\right)$. Histograms represent median values, and error bars refer to the interquartile range. post. cingulate, Posterior cingulate cortex; $\mathrm{PFC}$, prefrontal cortex.

revealed regional differences in their relative degrees (Fig. 3), indicating that additional biochemical mechanisms may amplify or attenuate hypometabolism or atrophy in specific brain regions.

The other notable finding is the regional variability in the hierarchy between the relative degrees of atrophy, hypometabolism, and $\mathrm{A} \beta$ deposition, which suggests that these alterations are subtended by multiple region-specific processes. Thus, in contrast to current global models that propose a general chronology in the appearance of the different alterations (independently of brain regions), the present study rather highlights the regional discrepancy in this sequence, suggesting that different subtending processes may be involved in the different brain regions. Although the absolute number of sets of brain regions is arbitrary because it depends on the subjective dendograms cutoff, our findings revealed clearly distinguishable patterns with three poles: the hippocampus, the posterior association areas, and the frontal cortex.

In the hippocampus, the presence of abnormally phosphorylated tau proteins that aggregate to form neurofibrillary tangles (NFTs) may be one of the processes underlying severe atrophy and moderate hypometabolism despite low A $\beta$ bur- den. Indeed, neurodegeneration seems to be closely related to tau pathology: progression of both gray matter atrophy (Whitwell et al., 2008) and hypoperfusion (Bradley et al., 2002) through the brain seems to follow NFT spreading as evaluated with Braak staging. In addition, neuronal loss has been shown to correlate with local counts of NFTs in different brain areas, such as the entorhinal cortex (Gómez-Isla et al., 1996) and superior temporal sulcus (Gómez-Isla et al., 1997). Consequently, because NFTs first appear and remain predominant in medial temporal structures as the disease pathology progresses (Braak and Braak, 1991; Delacourte et al., 1999), it is likely that NFTs, rather than $\mathrm{A} \beta$ deposition, are responsible for hippocampal atrophy and hypometabolism. However, the possibility of a distant effect of $\mathrm{A} \beta$ on the hippocampus cannot be ruled out, especially because the brain structures to which the hippocampus projects the most, i.e., the retrosplenial cortex and medial and orbital prefrontal cortices (Aggleton, 2012), are also those showing the highest $\mathrm{A} \beta$ load (Fig. 2, right).

In posterior association areas, several factors have been proposed to induce neuronal dysfunction over and above local A $\beta$ pathology. Notably, recent studies suggested that hypometabolism observed in patients with mild cognitive impairment and Alzheimer's disease dementia (especially in the posterior cingulate cortex) at least partly results from hippocampal atrophy through cingulate bundle disruption (Villain et al., 2008; Choo et al., 2010; Villain et al., 2010). Besides, as mentioned previously, relationships between hypometabolism in the precuneus and prefrontal A $\beta$ load have been observed (Cohen et al., 2009), possibly revealing distant $\mathrm{A} \beta$ effects. Actually, the posterior cingulate, precuneus, angular gyrus, and lateral temporal cortex are "cortical hubs" (Buckner et al., 2009), i.e., nodes in brain networks that are tightly connected to numerous brain structures. This may explain their vulnerability to Alzheimer's diseaserelated pathological processes in widely distributed brain regions.

Last, in frontal areas, $A \beta$ load reached its maxima, whereas atrophy and hypometabolism were minimal. Although already noticed by previous authors (Edison et al., 2007; Jack et al., 2008; Li et al., 2008), the present study provides a quantitative evidence for this discrepancy. This may appear surprising considering studies in animal models showing that severe neurite abnormalities especially develop in the vicinity of $\mathrm{A} \beta$ deposits (Tsai et al., 2004) because they are surrounded by a halo of soluble oligomeric forms of A $\beta$ (Koffie et al., 2009), themselves shown to be toxic to synapses (Lacor et al., 2007). Our findings may reflect (1) a lack of deleterious effect of local $\mathrm{A} \beta$ deposits in humans, (2) a difference in the timing of the different biomarkers (see above), or (3) the presence of compensation processes. This later hypothesis is supported by pre- 
vious functional MRI studies showing greater activations in patients with Alzheimer's disease relative to healthy controls (notably in the frontal cortex), positively associated with cognitive performances and interpreted as the reflect of compensation processes (for review, see Schwindt and Black, 2009). It is thus also possible that, faced with $A \beta$ deposition, neuronal and synaptic plasticity occurs, allowing to maintain neuronal integrity, glucose consumption, and brain volume.

Note that the patient sample size is relatively limited in the present study compared with large multicenter studies. However, (1) our patients have been thoroughly selected as having a high probability of Alzheimer's disease etiology based on neuroimaging biomarkers as recently recommended, (2) the use of multimodal data obtained in a single laboratory has clear advantage on multicenter data for complex and precise voxelwise analyses as performed here, (3) our findings were confirmed using nonparametric tests, and (4) our results are entirely consistent with previous studies when applicable. Thus, whole-brain patterns of atrophy, hypometabolism, and $\mathrm{A} \beta$ deposition (Fig. 2) are highly similar to those reported in studies from different samples (Buckner et al., 2005; Edison et al., 2007; Alsop et al., 2008; Chételat et al., 2008; Jack et al., 2008; Caroli et al., 2010; Fleisher et al., 2011), and the map of local mismatch between atrophy and hypometabolism (Fig. 3, left) is almost identical to previous studies by Alsop et al. (2008) and Caroli et al. (2010) despite the use of different methodologies and samples. However, we cannot exclude the possibility of a lack of statistical power in the analyses, for instance, to detect subtle relationships between $\mathrm{A} \beta$ deposition and neurodegeneration.

As a whole, our findings highlight the complex local relationships between atrophy, hypometabolism, and $\mathrm{A} \beta$ load and the regional heterogeneity in their hierarchy, suggesting the involvement of different underlying processes. It would be of particular interest to assess these relationships in earlier stages of the disease and to consider not only local but also distant phenomena to get a more comprehensive overview of these different brain alterations and their interactions. Finally, longitudinal studies are warranted to take into account the potential lapse between the different pathological processes while assessing their relationships and eventually propose a more complete, region-specific, model of Alzheimer's disease pathology evolution.

\section{References}

Aggleton JP (2012) Multiple anatomical systems embedded within the primate medial temporal lobe: implications for hippocampal function. Neurosci Biobehav Rev 36:1579-1596.

Alsop DC, Casement M, de Bazelaire C, Fong T, Press DZ (2008) Hippocampal hyperperfusion in Alzheimer's disease. Neuroimage 42: $1267-1274$.

Boccardi M, Laakso MP, Bresciani L, Galluzzi S, Geroldi C, Beltramello A, Soininen H, Frisoni GB (2003) The MRI pattern of frontal and temporal brain atrophy in fronto-temporal dementia. Neurobiol Aging 24:95-103.

Bourgeat P, Chételat G, Villemagne VL, Fripp J, Raniga P, Pike K, Acosta O, Szoeke C, Ourselin S, Ames D, Ellis KA, Martins RN, Masters CL, Rowe CC, Salvado O; AIBL Research Group (2010) Beta-amyloid burden in the temporal neocortex is related to hippocampal atrophy in elderly subjects without dementia. Neurology 74:121-127.

Braak H, Braak E (1991) Neuropathological stageing of Alzheimer-related changes. Acta Neuropathologica 82:239-259.

Bradley KM, O'Sullivan VT, Soper ND, Nagy Z, King EM, Smith AD, Shepstone BJ (2002) Cerebral perfusion SPET correlated with Braak pathological stage in Alzheimer's disease. Brain 125:1772-1781.

Buckner RL, Snyder AZ, Shannon BJ, LaRossa G, Sachs R, Fotenos AF, Sheline YI, Klunk WE, Mathis CA, Morris JC, Mintun MA (2005) Molecular, structural, and functional characterization of Alzheimer's disease: evi- dence for a relationship between default activity, amyloid, and memory. J Neurosci 25:7709-7717.

Buckner RL, Sepulcre J, Talukdar T, Krienen FM, Liu H, Hedden T, AndrewsHanna JR, Sperling RA, Johnson KA (2009) Cortical hubs revealed by intrinsic functional connectivity: mapping, assessment of stability, and relation to Alzheimer's disease. J Neurosci 29:1860-1873.

Camus V, Payoux P, Barré L, Desgranges B, Voisin T, Tauber C, La Joie R, Tafani M, Hommet C, Chételat G, Mondon K, de La Sayette V, Cottier JP, Beaufils E, Ribeiro MJ, Gissot V, Vierron E, Vercouillie J, Vellas B, Eustache F, Guilloteau D (2012) Using PET with (18)F-AV-45 (florbetapir) to quantify brain amyloid load in a clinical environment. Eur J Nucl Med Mol Imaging 39:621-631.

Caroli A, Lorenzi M, Geroldi C, Nobili F, Paghera B, Bonetti M, Cotelli M, Frisoni GB (2010) Metabolic compensation and depression in Alzheimer's disease. Dement Geriatr Cogn Disord 29:37-45.

Casanova R, Srikanth R, Baer A, Laurienti PJ, Burdette JH, Hayasaka S, Flowers L, Wood F, Maldjian JA (2007) Biological parametric mapping: a statistical toolbox for multimodality brain image analysis. Neuroimage 34:137-143.

Chételat G, Desgranges B, Landeau B, Mézenge F, Poline JB, de la Sayette V, Viader F, Eustache F, Baron JC (2008) Direct voxel-based comparison between grey matter hypometabolism and atrophy in Alzheimer's disease. Brain 131:60-71.

Chételat G, Villemagne VL, Bourgeat P, Pike KE, Jones G, Ames D, Ellis KA, Szoeke C, Martins RN, O'Keefe GJ, Salvado O, Masters CL, Rowe CC; Australian Imaging Biomarkers and Lifestyle Research Group (2010) Relationship between atrophy and beta-amyloid deposition in Alzheimer disease. Ann Neurol 67:317-324.

Choo IH, Lee DY, Oh JS, Lee JS, Lee DS, Song IC, Youn JC, Kim SG, Kim KW, Jhoo JH, Woo JI (2010) Posterior cingulate cortex atrophy and regional cingulum disruption in mild cognitive impairment and Alzheimer's disease. Neurobiol Aging 31:772-779.

Cohen AD, Price JC, Weissfeld LA, James J, Rosario BL, Bi W, Nebes RD, Saxton JA, Snitz BE, Aizenstein HA, Wolk DA, Dekosky ST, Mathis CA, Klunk WE (2009) Basal cerebral metabolism may modulate the cognitive effects of $A \beta$ in mild cognitive impairment: an example of brain reserve. J Neurosci 29:14770-14778.

Delacourte A, David JP, Sergeant N, Buée L, Wattez A, Vermersch P, Ghozali F, Fallet-Bianco C, Pasquier F, Lebert F, Petit H, Di Menza C (1999) The biochemical pathway of neurofibrillary degeneration in aging and Alzheimer's disease. Neurology 52:1158-1165.

Edison P, Archer HA, Hinz R, Hammers A, Pavese N, Tai YF, Hotton G, Cutler D, Fox N, Kennedy A, Rossor M, Brooks DJ (2007) Amyloid, hypometabolism, and cognition in Alzheimer disease: an $\left[{ }^{11} \mathrm{C}\right] \mathrm{PIB}$ and $\left[{ }^{18}\right.$ F]FDG PET study. Neurology 68:501-508.

Engler H, Forsberg A, Almkvist O, Blomquist G, Larsson E, Savitcheva I, Wall A, Ringheim A, Långström B, Nordberg A (2006) Two-year follow-up of amyloid deposition in patients with Alzheimer's disease. Brain 129: $2856-2866$

Ewers M, Sperling RA, Klunk WE, Weiner MW, Hampel H (2011) Neuroimaging markers for the prediction and early diagnosis of Alzheimer's disease dementia. Trends Neurosci 34:430-442.

Fagan AM, Head D, Shah AR, Marcus D, Mintun M, Morris JC, Holtzman DM (2009) Decreased cerebrospinal fluid Abeta(42) correlates with brain atrophy in cognitively normal elderly. Ann Neurol 65:176-183.

Fleisher AS, Chen K, Liu X, Roontiva A, Thiyyagura P, Ayutyanont N, Joshi AD, Clark CM, Mintun MA, Pontecorvo MJ, Doraiswamy PM, Johnson KA, Skovronsky DM, Reiman EM (2011) Using positron emission tomography and florbetapir $\mathrm{f} 18$ to image cortical amyloid in patients with mild cognitive impairment or dementia due to Alzheimer disease. Arch Neurol 68:1404-1411.

Forsberg A, Almkvist O, Engler H, Wall A, Långström B, Nordberg A (2010) High PIB retention in Alzheimer's disease is an early event with complex relationship with CSF biomarkers and functional parameters. Curr Alzheimer Res 7:56-66.

Furst AJ, Rabinovici GD, Rostomian AH, Steed T, Alkalay A, Racine C, Miller BL, Jagust WJ (2012) Cognition, glucose metabolism and amyloid burden in Alzheimer's disease. Neurobiol Aging 33:215-225.

Gómez-Isla T, Price JL, McKeel DW Jr, Morris JC, Growdon JH, Hyman BT (1996) Profound loss of layer II entorhinal cortex neurons occurs in very mild Alzheimer's disease. J Neurosci 16:4491-4500.

Gómez-Isla T, Hollister R, West H, Mui S, Growdon JH, Petersen RC, Parisi 
JE, Hyman BT (1997) Neuronal loss correlates with but exceeds neurofibrillary tangles in Alzheimer's disease. Ann Neurol 41:17-24.

Hardy J, Selkoe DJ (2002) The amyloid hypothesis of Alzheimer's disease: progress and problems on the road to therapeutics. Science 297:353-356.

Herholz K, Ebmeier K (2011) Clinical amyloid imaging in Alzheimer's disease. Lancet Neurol 10:667-670.

Hyman BT (2011) Amyloid-dependent and amyloid-independent stages of Alzheimer disease. Arch Neurol 68:1062-1064.

Jack CR Jr, Petersen RC, Xu YC, Waring SC, O’Brien PC, Tangalos EG, Smith GE, Ivnik RJ, Kokmen E (1997) Medial temporal atrophy on MRI in normal aging and very mild Alzheimer's disease. Neurology 49:786-794.

Jack CR Jr, Lowe VJ, Senjem ML, Weigand SD, Kemp BJ, Shiung MM, Knopman DS, Boeve BF, Klunk WE, Mathis CA, Petersen RC (2008) 11C PiB and structural MRI provide complementary information in imaging of Alzheimer's disease and amnestic mild cognitive impairment. Brain 131:665-680.

Jack CR Jr, Knopman DS, Jagust WJ, Shaw LM, Aisen PS, Weiner MW, Petersen RC, Trojanowski JQ (2010) Hypothetical model of dynamic biomarkers of the Alzheimer's pathological cascade. Lancet Neurol 9:119-128.

Koffie RM, Meyer-Luehmann M, Hashimoto T, Adams KW, Mielke ML, Garcia-Alloza M, Micheva KD, Smith SJ, Kim ML, Lee VM, Hyman BT, Spires-Jones TL (2009) Oligomeric amyloid beta associates with postsynaptic densities and correlates with excitatory synapse loss near senile plaques. Proc Natl Acad Sci U S A 106:4012-4017.

Lacor PN, Buniel MC, Furlow PW, Clemente AS, Velasco PT, Wood M, Viola KL, Klein WL (2007) A $\beta$ oligomer-induced aberrations in synapse composition, shape, and density provide a molecular basis for loss of connectivity in Alzheimer's disease. J Neurosci 27:796-807.

Li Y, Rinne JO, Mosconi L, Pirraglia E, Rusinek H, DeSanti S, Kemppainen N, Någren K, Kim BC, Tsui W, de Leon MJ (2008) Regional analysis of FDG and PIB-PET images in normal aging, mild cognitive impairment, and Alzheimer's disease. Eur J Nucl Med Mol Imaging 35:2169-2181.

McKhann GM, Knopman DS, Chertkow H, Hyman BT, Jack CR Jr, Kawas CH, Klunk WE, Koroshetz WJ, Manly JJ, Mayeux R, Mohs RC, Morris JC, Rossor MN, Scheltens P, Carrillo MC, Thies B, Weintraub S, Phelps CH (2011) The diagnosis of dementia due to Alzheimer's disease: recommendations from the National Institute on Aging-Alzheimer's Association workgroups on diagnostic guidelines for Alzheimer's disease. Alzheimers Dement 7:263-269.

McKhann G, Drachman D, Folstein M, Katzman R, Price D, Stadlan EM (1984) Clinical diagnosis of Alzheimer's disease: report of the NINCDSADRDA Work Group under the auspices of Department of Health and Human Services Task Force on Alzheimer's Disease. Neurology 34:939-944.

Palop JJ, Mucke L (2010) Amyloid- $\beta$-induced neuronal dysfunction in Alzheimer's disease: from synapses toward neural networks. Nat Neurosci 13:812-818.

Parihar MS, Brewer GJ (2010) Amyloid- $\beta$ as a modulator of synaptic plasticity. J Alzheimers Dis 22:741-763.

Perrin RJ, Fagan AM, Holtzman DM (2009) Multimodal techniques for diagnosis and prognosis of Alzheimer's disease. Nature 461:916-922.
Rabinovici GD, Roberson ED (2010) Beyond diagnosis: what biomarkers are teaching us about the "biology" of Alzheimer disease. Ann Neurol 67:283-285

Rabinovici GD, Furst AJ, Alkalay A, Racine CA, O’Neil JP, Janabi M, Baker SL, Agarwal N, Bonasera SJ, Mormino EC, Weiner MW, Gorno-Tempini ML, Rosen HJ, Miller BL, Jagust WJ (2010) Increased metabolic vulnerability in early-onset Alzheimer's disease is not related to amyloid burden. Brain 133:512-528.

Richardson MP, Friston KJ, Sisodiya SM, Koepp MJ, Ashburner J, Free SL, Brooks DJ, Duncan JS (1997) Cortical grey matter and benzodiazepine receptors in malformations of cortical development. A voxel-based comparison of structural and functional imaging data. Brain 120:1961-1973.

Rowe CC, Ellis KA, Rimajova M, Bourgeat P, Pike KE, Jones G, Fripp J, Tochon-Danguy H, Morandeau L, O'Keefe G, Price R, Raniga P, Robins P, Acosta O, Lenzo N, Szoeke C, Salvado O, Head R, Martins R, Masters CL, Ames D, Villemagne VL (2010) Amyloid imaging results from the Australian Imaging, Biomarkers and Lifestyle (AIBL) study of aging. Neurobiol Aging 31:1275-1283.

Schroeter ML, Stein T, Maslowski N, Neumann J (2009) Neural correlates of Alzheimer's disease and mild cognitive impairment: a systematic and quantitative meta-analysis involving 1351 patients. Neuroimage 47: 1196-1206.

Schwindt GC, Black SE (2009) Functional imaging studies of episodic memory in Alzheimer's disease: a quantitative meta-analysis. Neuroimage 45:181-190.

Sperling RA, Aisen PS, Beckett LA, Bennett DA, Craft S, Fagan AM, Iwatsubo T, Jack CR Jr, Kaye J, Montine TJ, Park DC, Reiman EM, Rowe CC, Siemers E, Stern Y, Yaffe K, Carrillo MC, Thies B, Morrison-Bogorad M, Wagster MV, Phelps CH (2011) Toward defining the preclinical stages of Alzheimer's disease: recommendations from the National Institute on Aging and the Alzheimer's Association workgroup. Alzheimers Dement 7:280-292.

Tsai J, Grutzendler J, Duff K, Gan WB (2004) Fibrillar amyloid deposition leads to local synaptic abnormalities and breakage of neuronal branches. Nat Neurosci 7:1181-1183.

Tzourio-Mazoyer N, Landeau B, Papathanassiou D, Crivello F, Etard O, Delcroix N, Mazoyer B, Joliot M (2002) Automated anatomical labeling of activations in SPM using a macroscopic anatomical parcellation of the MNI MRI single-subject brain. Neuroimage 15:273-289.

Villain N, Desgranges B, Viader F, de la Sayette V, Mézenge F, Landeau B, Baron JC, Eustache F, Chételat G (2008) Relationships between hippocampal atrophy, white matter disruption, and gray matter hypometabolism in Alzheimer's disease. J Neurosci 28:6174-6181.

Villain N, Fouquet M, Baron JC, Mézenge F, Landeau B, de La Sayette V, Viader F, Eustache F, Desgranges B, Chételat G (2010) Sequential relationships between grey matter and white matter atrophy and brain metabolic abnormalities in early Alzheimer's disease. Brain 133:3301-3314.

Whitwell JL, Josephs KA, Murray ME, Kantarci K, Przybelski SA, Weigand SD, Vemuri P, Senjem ML, Parisi JE, Knopman DS, Boeve BF, Petersen RC, Dickson DW, Jack CR Jr (2008) MRI correlates of neurofibrillary tangle pathology at autopsy. Neurology 71:743-749. 